\title{
Effect of the Fe Nanoparticles Generated by Pulsed Plasma in Liquid in the Catalyzed Ozone Removal of Phenolphthalein
}

\author{
O. Olea-Mejia, ${ }^{1}$ M. Fernández-Mondragón, ${ }^{1}$ C. Barrera-Díaz, ${ }^{1}$ and A. Cabral-Prieto ${ }^{2}$ \\ ${ }^{1}$ Centro Conjunto de Investigación en Química Sustentable (CCIQS) UAEM-UNAM, Universidad Autónoma del Estado de México, \\ Carr. Toluca-Atlacomulco km 14.5, 50200 Toluca, MEX, Mexico \\ ${ }^{2}$ Departamento de Física, Instituto Nacional de Investigaciones Nucleares, Apdo. Postal 18-1027, Mexico City, Mexico
}

Correspondence should be addressed to O. Olea-Mejia; oleaoscar@yahoo.com.mx

Received 26 December 2016; Accepted 31 January 2017; Published 28 March 2017

Academic Editor: Leonardo Palmisano

Copyright ( 2017 O. Olea-Mejia et al. This is an open access article distributed under the Creative Commons Attribution License, which permits unrestricted use, distribution, and reproduction in any medium, provided the original work is properly cited.

We have synthesized, in this work, zero valent iron (ZVI) nanoparticles to improve the efficiency of degradation of phenolphthalein catalyzed by ozone in aqueous solution. The Fe nanoparticles were obtained using the pulsed plasma in liquid (PPL) method with water as the liquid medium. Such nanoparticles have a mean size of $12 \mathrm{~nm}$ and are composed of $\sim 80 \% \mathrm{Fe}^{0}$, while the rest are a mixture of $\mathrm{Fe}^{+2}$ and $\mathrm{Fe}^{+3}$ oxides. The degradation of phenolphthalein was carried on a glass reactor injecting a constant amount of ozone and introducing different concentrations of Fe nanoparticles to the system. When using pure ozone, the percentage of degradation of phenolphthalein measured by colorimetry after one hour of reaction was $84 \%$. However, when Fe nanoparticles are used, such percentage can be as high as $98 \%$ in 50 minutes of reaction. Furthermore, the degradation rate constant was $0.0334 \mathrm{~min}^{-1}$ with only ozone and it can be as high as $0.0733 \mathrm{~min}^{-1}$ with Fe nanoparticles. Finally, the total mineralization of phenolphthalein was obtained by total organic carbon (TOC) determinations. It is shown that when using only ozone, we obtained a percentage of mineralization of $49 \%$ and $96 \%$ when using the highest concentration of Fe nanoparticles.

\section{Introduction}

Because of the increasing human activity, wastewaters have become a high-priority issue since the produced contaminants can reach oceans, rivers, underground water, and agricultural soil. This results in population exposure to hazardous substances that lead to public health threat. Among these dangerous substances, we find organic molecules that can be very toxic, carcinogenic, and difficult to eliminate. For these contaminants, it is not enough to use conventional wastewater techniques such as biological, adsorptive, or partial decomposition, since the intermediate products are sometimes worse than those of the original molecule.

One of the novel ways to treat water containing biorefractory or difficult to remove compounds is by using advanced oxidation processes (AOPs), which depends on the hydroxyl radical formation. This radical $(\bullet \mathrm{OH})$ is considered highly reactive, a powerful oxidant, which is easily formed and ready to react leading harmless products [1].
AOPs are an attractive alternative to reach complete degradation (mineralization) of organic molecules to $\mathrm{H}_{2} \mathrm{O}, \mathrm{CO}_{2}$, and inorganic salts. The Fenton reaction is a well-known AOP that has been used for many decades and is still under studies because of low-cost and low-temperature and pressure operation [2]. In this reaction, the production of the very oxidative $\bullet \mathrm{OH}$ radicals is the key to degrade the organic molecules. However, the traditional Fenton reaction requires controlling the aqueous $\mathrm{pH}$, indeed the best value for this reaction to proceed is 2.8 . This condition limits the use of this reaction. Therefore, to produce better results, this reaction can be assisted and one way to do it is by applying light to enhance the production of $\bullet \mathrm{OH}$ radicals. Nevertheless, light introduction is not an easy procedure and is limited by its region action [3-6].

A novel way to improve the Fenton reaction is by introducing ozone to the system which can tackle the degradation issue by two approaches: first, ozone itself can directly oxidize organic molecules and second, it also can react with iron in solution to produce the desired hydroxyl radicals $[7,8]$. 


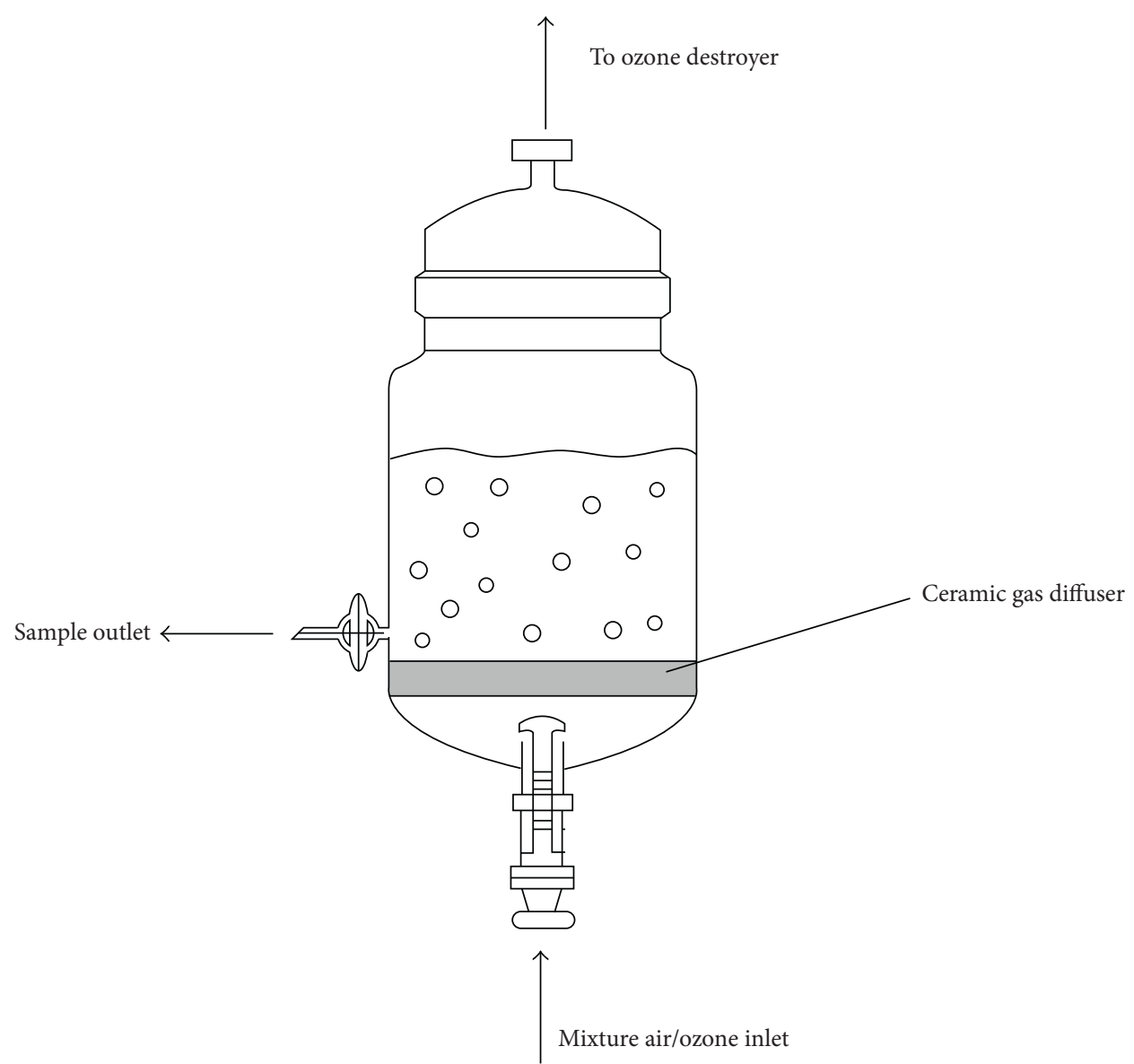

FIGURE 1: Schematics of the reactor used in the phenolphthalein degradation experiments.

In the last decade, Fe-containing compounds in the nanometric scale have been used in the reactions mentioned above $[2-4,7,9,10]$. The advantage of using these materials is the very high surface area available for the catalytic activity. More recently, zero valent iron (ZVI) nanoparticles have been employed to further improve the efficiency of the oxidation process $[6,11-16]$. Such nanoparticles have been produced mainly using chemical methods, which have the disadvantage to use expensive and/or toxic reagents and to produce undesirable by-products.

To overcome this problem, in this work, we propose the use of pulsed plasma in liquid to produce pure, uncovered ZVI nanoparticles and use them in the degradation of phenolphthalein, which is widely used as an acidic-basic indicator, and because of its intense color at low concentrations, it is a suitable model molecule to easily follow its degradation. By using this method, we avoid the presence of any coating on the particle surface increasing the efficiency of the AOP because the $\mathrm{Fe}$ atoms are readily exposed to the molecules involved in the oxidation process [17-22]. There are also no by-products that could interfere with the different reactions happening during the AOP. When we couple the ozone-Fe nanoparticle system, we improve the mass transfer in the system, which results in a faster process able to react in a wide range of $\mathrm{pH}$.

\section{Materials and Methods}

2.1. Zero Valent Iron (ZVI) Nanoparticle Synthesis and Characterization. As mentioned, the method used in this work to produce ZVI nanoparticles was pulsed plasma in liquid (PPL). Briefly, 40 A of AC current is applied to two rods of iron $(15 \times 4 \times 3 \mathrm{~mm}, 99 \%$ of purity) immersed in $20 \mathrm{~mL}$ of distilled water. One of the rods is still while the other is oscillating to achieve intermittent contact between them. The time of the experiment varied according to the amount of nanoparticles desired, and it varied from 3 to 10 minutes. The obtained nanostructures were characterized by transmission electron microscopy (TEM) to observe morphology and crystal structure with a JEOL JEM-2100 microscope at $200 \mathrm{keV}$ of acceleration voltage. Mossbauer spectroscopy (MS) was used to determine the chemical state of the $\mathrm{Fe}$ atoms before and after the oxidation experiments with a Wissel Mossbauer spectrometer coupled to a ${ }^{57} \mathrm{Co} / \mathrm{Rh}$ source in the constant acceleration mode; for these measurements, the particles were encapsulated inside an epoxy resin to avoid oxidation with air.

2.2. Degradation of Phenolphthalein. A $2 \mathrm{~L}$ glass reactor was used for the experiments (Figure 1). This reactor has a ceramic gas diffusor and a gas inlet at the bottom. The gas 


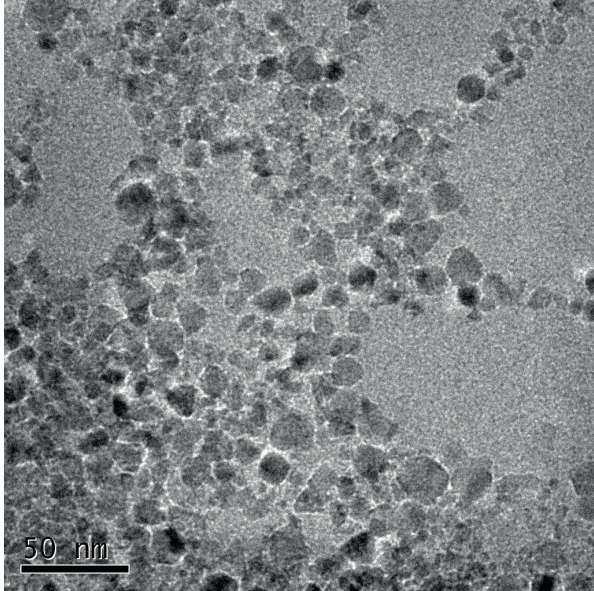

(a)

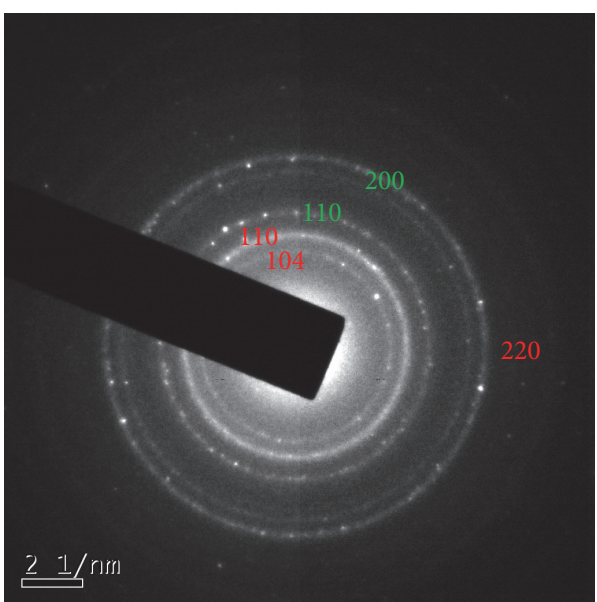

(b)

Figure 2: (a) TEM image of the ZVI nanoparticles. (b) Diffraction pattern of ZVI nanoparticles, the green Miller indexes are for metallic iron and the red indexes are for iron oxide.

flow exits from the top of a reactor and enters an ozone destroyer to eliminate all unreacted ozone. The gas introduced to the reactor was a 75/25 air/ozone mixture at a constant ozone concentration of $5 \pm 0.5 \mathrm{mg} / \mathrm{L}$ for all experiments. The equipment used to produce the ozone was a Pacific Ozone Technology generator number LAB212.

$500 \mathrm{~mL}$ of water solution $(60 \mathrm{ppm})$ of phenolphthalein was introduced to the reactor, and the $\mathrm{pH}$ was adjusted to 3 with $\mathrm{H}_{2} \mathrm{SO}_{4} 1 \mathrm{M}$. Then, the ozone was injected and immediately after, the nanoparticles were introduced. We used three different amounts of ZVI nanoparticles: 30, 60, and $90 \mathrm{mg}$ as well as only ozone for comparison. The reaction was monitored for 60 minutes taking a small sample $(2 \mathrm{~mL})$ each 10 minutes to monitor the phenolphthalein degradation. The phenolphthalein concentration was measured by light absorption. Each sample taken from the reactor was adjusted to a $\mathrm{pH}$ of 13 with a solution of $\mathrm{NaOH} 1 \mathrm{M}$ to achieve the characteristic purple color of phenolphthalein in basic solution and measured with a HACH DR/5000 Uv-Vis spectrophotometer at a wavelength of $550 \mathrm{~nm}$. Total organic carbon (TOC) was measured with a TOC-LCPH/CPN Shimadzu total organic carbon analyzer after the end of each experiment to study the mineralization of the phenolphthalein. Each experiment was repeated three times, and the results presented are the average of the three measurements.

\section{Results and Discussion}

3.1. ZVI Nanoparticle Characterization. Figure 2 shows the TEM image of the ZVI nanoparticles. As seen, the particles are roughly spherical and the mean size is $12 \mathrm{~nm}$ and the indexation of the diffraction pattern (Figure 2(b)) shows that they are made of a mixture of zero valent iron and iron oxide. It is important to have small particles composed of $\mathrm{Fe}^{0}$ atoms to improve the formation of $\bullet \mathrm{OH}$ ions. The increase in production of such ions is due to the availability of Fe atoms on the surface of small particles (high surface area) and the fact that Fe atoms are in the metallic state which means that there are more electrons available per atom to produce the - OH ions which will be discussed later. However, the iron oxide seen in Figure 2(b) could be formed during the sample preparation of the TEM samples, since it was done in air and the small amount of particles on the TEM grid is readily exposed to oxygen from air. To avoid oxidation of particles during their characterization and to better study the Fe oxidation state of the particles in the solution, they were studied by Mossbauer spectroscopy and the results are shown in Figure 3(a). Observing the spectrum, it is evident that it is composed of more than one signal and a signal deconvolution is necessary. By doing this, we can identify three signals with different isomeric shifts. The first one is a sextet corresponding to $\mathrm{Fe}^{0}$, while the other two signals come from $\mathrm{Fe}^{+2}$ and $\mathrm{Fe}^{+3}$ ions as evidenced by the corresponding isomeric shift for each species $\left(0.10 \mathrm{~mm} / \mathrm{s}\right.$ for $\mathrm{Fe}^{+3}$ and $0.42 \mathrm{~mm} / \mathrm{s}$ for $\mathrm{Fe}^{+2}$ ). Their respective abundance in the sample is $80.2 \%, 4.5 \%$, and $15.3 \%$ for $\mathrm{Fe}^{0}, \mathrm{Fe}^{+2}$, and $\mathrm{Fe}^{+3}$, respectively. From these results, we can conclude that there are some iron oxide present formed during the particle synthesis; however, the majority of the Fe atoms are in the metallic state.

3.2. Oxidative Degradation of Phenolphthalein. The percentage of degradation of phenolphthalein against time is presented in Figure 4 for all the experiments. First, we will focus on the process with only ozone as oxidative agent. As it can be seen, by the first 30 minutes of reaction, the degradation of the molecule reached more than $50 \%$ and at 60 minutes, it was $84 \%$. The degradation with pure ozone has been studied, and its mechanism can be explained with several possible simultaneous chemical reactions:

$$
\begin{aligned}
2 \mathrm{O}_{3}+\mathrm{H}_{2} \mathrm{O} & \rightarrow \mathrm{OH} \bullet+2 \mathrm{O}_{2}+\mathrm{HO}_{2} \bullet \\
\mathrm{O}_{3}+3 \mathrm{H}_{2} \mathrm{O} & \rightarrow 3 \mathrm{H}_{2} \mathrm{O}_{2} \\
\mathrm{H}_{2} \mathrm{O}_{2} & \rightarrow \mathrm{HO}_{2}^{\bullet}+\mathrm{H}^{+}
\end{aligned}
$$




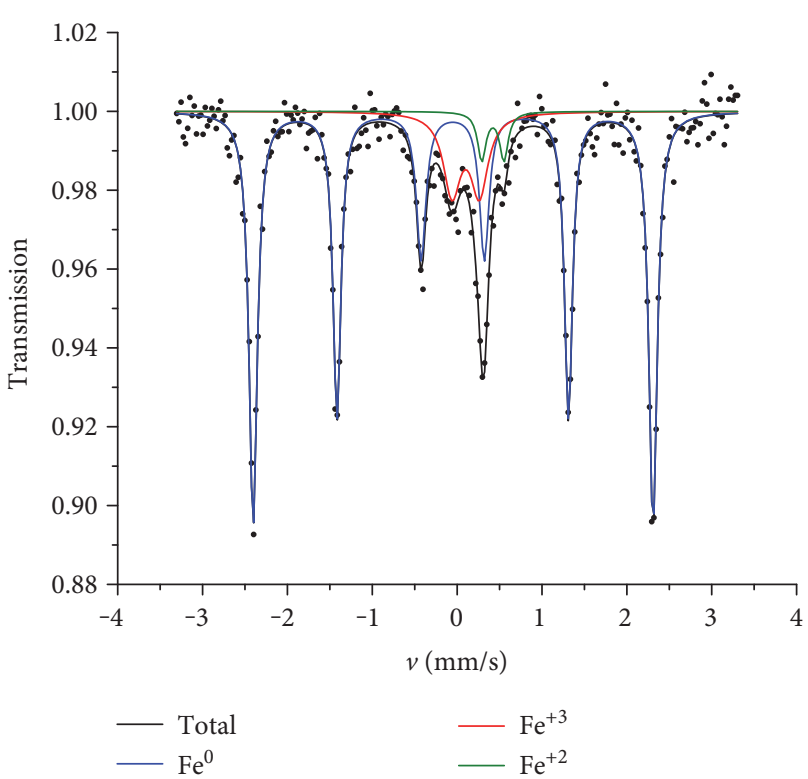

(a)

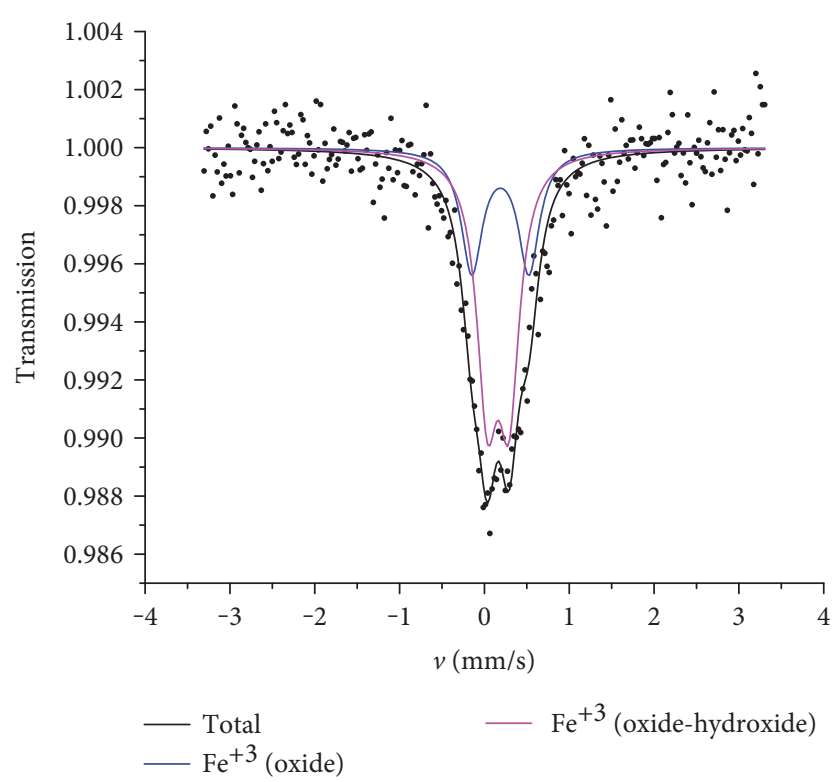

(b)

Figure 3: Mossbauer spectroscopy of the Fe nanoparticles (a) before the phenolphthalein degradation experiments and (b) after the phenolphthalein degradation experiments.

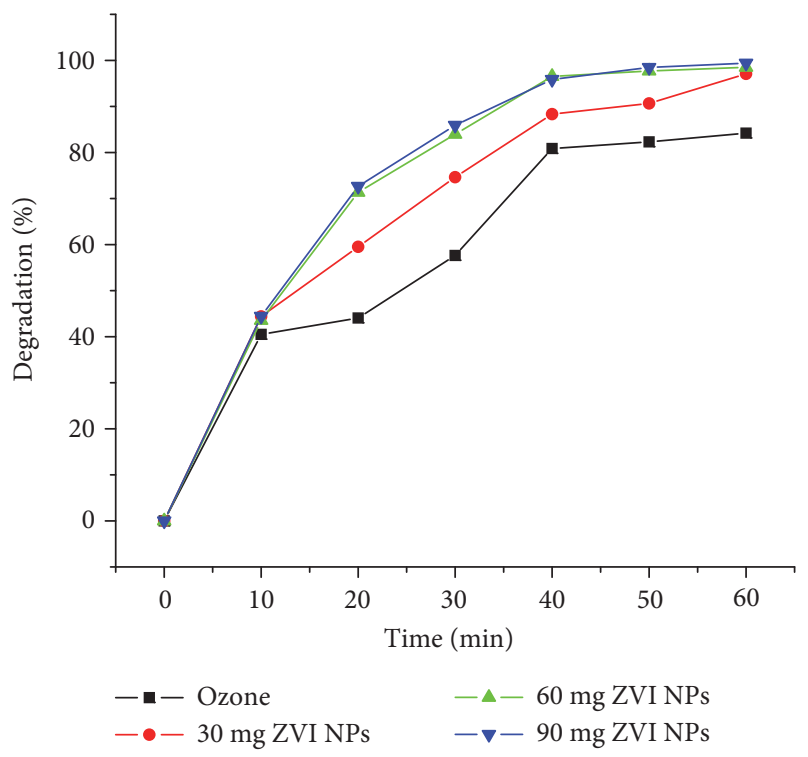

Figure 4: Percentage of degradation of phenolphthalein with different doses of ZVI nanoparticles.

Once the $\mathrm{H}_{2} \mathrm{O}_{2}$ is formed, the peroxonation process is also plausible to happen:

$$
\begin{aligned}
& \mathrm{H}_{2} \mathrm{O}_{2} \leftrightarrow \mathrm{HO}_{2}^{-}+\mathrm{H}^{+} \\
& \mathrm{HO}_{2}^{-}+\mathrm{O}_{3} \leftrightarrow \mathrm{HO}_{2}{ }^{-}+\mathrm{O}_{3}^{\bullet-} \\
& \mathrm{O}_{3}{ }^{--}+\mathrm{H}^{+} \leftrightarrow \mathrm{HO}_{3} \rightarrow \mathrm{OH} \bullet+\mathrm{O}_{2}
\end{aligned}
$$

From these reactions, we can observe that pure ozone is capable of forming the oxidative $\bullet \mathrm{OH}$ radical and therefore the observed degradation of phenolphthalein.

On the other hand, also from Figure 4, we see that when ZVI nanoparticles are incorporated to the system, the achieved degradation is higher and occurs in less time compared to that of pure ozone. For $30 \mathrm{mg}$ of Fe nanoparticles, the final degradation percentage was $97 \%$. In the case of $60 \mathrm{mg}$ and $90 \mathrm{mg}$ of $\mathrm{Fe}$ nanoparticles, the final degradation was $97 \%$ and $98 \%$, respectively; however, this value was reached 10 minutes faster $(50 \mathrm{~min})$. It is very clear that the addition of ZVI nanoparticles contributes greatly to the degradation of phenolphthalein. And this can be explained with the following reactions:

$$
\begin{aligned}
& \mathrm{Fe}^{0}+2 \mathrm{O}_{3} \rightarrow 2 \mathrm{O}_{3}^{\cdot-}+\mathrm{Fe}^{+2} \\
& \mathrm{Fe}^{+2}+\mathrm{O}_{3} \rightarrow \mathrm{O}_{3}^{\cdot-}+\mathrm{Fe}^{+3} \\
& \mathrm{O}_{3}^{\cdot-}+\mathrm{H}^{+} \rightarrow \mathrm{O}_{2}+\mathrm{OH} \bullet
\end{aligned}
$$

Equations (8) and (9) are typical of the common Fenton reaction in acidic medium. However, when $\mathrm{Fe}^{0}$ atoms are introduced to the system, there are two extra electrons per $\mathrm{Fe}$ atom to form the $\mathrm{OH} \bullet$ radical as seen in (7). To prove the conversion from $\mathrm{Fe}^{0}$ to $\mathrm{Fe}^{+3}$, Mossbauer spectroscopy was done to the particles after 60 minutes of reaction and it is shown in Figure 3(b). As seen, the isomeric shift of the two present signals corresponds to $\mathrm{Fe}^{+3}$ species that are from the mixture of iron oxide and iron oxide-hydroxide.

Since not only the final percentage of degradation is affected but the reaction time too, we followed the kinetics of the reaction with the following equation: 
TAble 1: Phenolphthalein degradation rate constants and TOC results.

\begin{tabular}{lcc}
\hline & $\begin{array}{c}\text { Degradation rate } \\
\text { constant }\left(\mathrm{min}^{-1}\right)\end{array}$ & $\begin{array}{c}\text { \% of TOC } \\
\text { removed }\end{array}$ \\
\hline Ozone only & 0.0334 & 49 \\
$30 \mathrm{mg}$ ZVI NPs & 0.0441 & 86 \\
$60 \mathrm{mg}$ ZVI NPs & 0.0721 & 89 \\
$90 \mathrm{mg}$ ZVI NPs & 0.0733 & 96 \\
\hline
\end{tabular}

$$
\frac{d C_{\mathrm{Ph}}}{d t}=-r_{\mathrm{Ph}}=K^{\prime} C_{\mathrm{Ph}}^{\alpha} C_{\mathrm{oz}}^{\beta}
$$

where $d C_{\mathrm{Ph}} / d t=r_{\mathrm{Ph}}=$ degradation rate of phenolphthalein, $K^{\prime}=$ rate constant, $C_{\mathrm{Ph}}=$ concentration of phenolphthalein, $\alpha=$ order of the reaction of phenolphthalein, $C_{\mathrm{oz}}=$ concentration of ozone, and $\beta=$ order of the reaction of ozone.

By considering the concentration of ozone constant throughout the experiment because of its abundance compared to that of the other reactants, we have

$$
-r_{\mathrm{Ph}}=K C_{\mathrm{Ph}}^{\alpha}
$$

Then, we consider a first order reaction $(\alpha=1)$ and solving for $C_{\mathrm{Ph}}$, we have

$$
\ln \left(\frac{C_{\mathrm{Ph}_{o}}}{C_{\mathrm{Ph}}}\right)=K t
$$

We have used (12) for all experiments, and the correlation index $R^{2}$ was more than 0.99 in all cases, confirming the first order behavior of our system. The results from this study are summarized in Table 1 . We can see that the reaction rate is increased as we increase the amount of ZVI nanoparticles, and it can be doubled when using $90 \mathrm{mg}$ of iron nanoparticles compared with the use of pure ozone. The reason for this increase in the reaction rate is due to the higher production of $\bullet \mathrm{OH}$ radicals according to the reactions already shown.

The colorimetric method used to determine the concentration of phenolphthalein is applied only to measure the degradation of the molecule. However, it is impossible to know the extent to complete mineralization. For this purpose, we performed measurements of total organic carbon (TOC) at the end of each experiment and the results are shown in Table 1. As seen, the addition of ZVI nanoparticles not only accelerates the degradation of phenolphthalein but also increases the extent of its complete mineralization. When using pure ozone, the organic carbon left in the solution is $51 \%$ of the original amount at the beginning of the reaction. This organic carbon can be in the form of any degradation products such as phenols and so forth which can be even more toxic than the original molecule. On the other hand, when using $90 \mathrm{mg}$ of $\mathrm{Fe}$ nanoparticles, the remaining organic carbon is only $4 \%$, which means that $96 \%$ of the original organic carbon is transformed into $\mathrm{H}_{2} \mathrm{O}+\mathrm{CO}_{2}$.
In previous works, we have studied two methods for the degradation of phenolphthalein in an aqueous solution. The first one is an electrochemical method which requires acidic conditions ( $\mathrm{pH}$ around 3) to reach a $71 \%$ Chemical Oxygen Demand (COD) removal. The other one is the use of ozone which needs basic conditions to obtain a 25\% COD removal. This difference in results can be explained in terms of the hydroxyl radical production, which is faster in the electrochemical methods; furthermore, less reactions are required to produce the radicals as compared to those with the use of ozonation [23].

\section{Conclusions}

We have synthesized zero valent iron nanoparticles using the pulsed plasma in liquid method. The nanoparticle mean diameter is $12 \mathrm{~nm}$ having the majority of their Fe atoms in the metallic phase as evidenced by Mossbauer spectroscopy. We have used these nanoparticles to enhance the degradation of phenolphthalein by ozonation at different particle concentrations. When using only ozone, the degradation measured by colorimetry was $84 \%$ at 60 minutes; however, when ZVI nanoparticles are introduced to the reactor, the degradation is as high as $98 \%$ with $90 \mathrm{mg}$ of NPs. The degradation rate constant was also calculated, and it was found that when using Fe nanoparticles, the value of this constant can be twice as big as the one obtained with only ozone. Finally, the incorporation of $\mathrm{Fe}$ to the system leads to an almost complete mineralization (96\%) of phenolphthalein while it is only $49 \%$ for only ozone.

\section{Conflicts of Interest}

The authors declare that they have no conflicts of interest.

\section{References}

[1] M. Munoz, Z. M. de Pedro, J. A. Casas, and J. J. Rodriguez, "Preparation of magnetite-based catalysts and their application in heterogeneous Fenton oxidation - a review," Applied Catalysis B: Environmental, vol. 176-177, pp. 249-265, 2015.

[2] R. Davarnejad and J. Azizi, "Alcoholic wastewater treatment using electro-Fenton technique modified by $\mathrm{Fe} 2 \mathrm{O} 3$ nanoparticles," Journal of Environmental Chemical Engineering, vol. 4, no. 2, pp. 2342-2349, 2016.

[3] F. Chai, K. Li, C. Song, and X. Guo, "Synthesis of magnetic porous $\mathrm{Fe} 3 \mathrm{O} 4 / \mathrm{C} / \mathrm{Cu} 2 \mathrm{O}$ composite as an excellent photoFenton catalyst under neutral condition," Journal of Colloid and Interface Science, vol. 475, pp. 119-125, 2016.

[4] L. M. Pastrana-Martínez, N. Pereira, R. Lima, J. L. Faria, H. T. Gomes, and A. M. T. Silva, "Degradation of diphenhydramine by photo-Fenton using magnetically recoverable iron oxide nanoparticles as catalyst," Chemical Engineering Journal, vol. 261, pp. 45-52, 2015.

[5] J. G. Mahy, L. Tasseroul, A. Zubiaur et al., "Highly dispersed iron xerogel catalysts for p-nitrophenol degradation by photo-Fenton effects," Microporous and Mesoporous Materials, vol. 197, pp. 164-173, 2014. 
[6] G. B. Ortiz de la Plata, O. M. Alfano, and A. E. Cassano, "2-Chlorophenol degradation via photo Fenton reaction employing zero valent iron nanoparticles," Journal of Photochemistry and Photobiology A: Chemistry, vol. 233, pp. 53-59, 2012.

[7] A. Ziylan and N. H. Ince, "Catalytic ozonation of ibuprofen with ultrasound and Fe-based catalysts," Catalysis Today, Part A, vol. 240, pp. 2-8, 2015.

[8] T. Torres-Blancas, G. Roa-Morales, C. Barrera-Díaz et al., "Ozonation of indigo carmine enhanced by Fe/Pimenta dioica L. Merrill particles," International Journal of Photoenergy, vol. 2015, Article ID 608412, p. 9, 2015.

[9] Y. Wang, H. Zhao, and G. Zhao, "Iron-copper bimetallic nanoparticles embedded within ordered mesoporous carbon as effective and stable heterogeneous Fenton catalyst for the degradation of organic contaminants," Applied Catalysis B: Environmental, vol. 164, pp. 396-406, 2015.

[10] S. Shin, H. Yoon, and J. Jang, "Polymer-encapsulated iron oxide nanoparticles as highly efficient Fenton catalysts," Catalysis Communications, vol. 10, no. 2, pp. 178-182, 2008.

[11] R. Cheng, C. Cheng, G.-h. Liu, X. Zheng, G. Li, and J. Li, "Removing pentachlorophenol from water using a nanoscale zero-valent iron/H2O2 system," Chemosphere, vol. 141, pp. 138-143, 2015.

[12] S. Machado, W. Stawiński, P. Slonina et al., "Application of green zero-valent iron nanoparticles to the remediation of soils contaminated with ibuprofen," Science of the Total Environment, vol. 461-462, pp. 323-329, 2013.

[13] T. Shahwan, S. Abu Sirriah, M. Nairat et al., "Green synthesis of iron nanoparticles and their application as a Fenton-like catalyst for the degradation of aqueous cationic and anionic dyes," Chemical Engineering Journal, vol. 172, no. 1, pp. 258-266, 2011.

[14] R. Chand, N. H. Ince, P. R. Gogate, and D. H. Bremner, "Phenol degradation using 20, 300 and $520 \mathrm{kHz}$ ultrasonic reactors with hydrogen peroxide, ozone and zero valent metals," Separation and Purification Technology, vol. 67, no. 1, pp. 103-109, 2009.

[15] D. H. Bremner, A. E. Burgess, D. Houllemare, and K. C. Namkung, "Phenol degradation using hydroxyl radicals generated from zero-valent iron and hydrogen peroxide," Applied Catalysis B: Environmental, vol. 63, no. 1, pp. 15-19, 2006.

[16] P. Raja, A. Bozzi, W. F. Jardim, G. Mascolo, R. Renganathan, and J. Kiwi, "Reductive/oxidative treatment with superior performance relative to oxidative treatment during the degradation of 4-chlorophenol," Applied Catalysis B: Environmental, vol. 59, no. 3, pp. 249-257, 2005.

[17] L. Chen, T. Mashimo, C. Iwamoto et al., "Synthesis of novel CoCx@C nanoparticles,” Nanotechnology, vol. 24, p. 045602, 2013.

[18] Z. Abdullaeva, E. Omurzak, C. Iwamoto et al., "High temperature stable WC1-x@C and TiC@C core-shell nanoparticles by pulsed plasma in liquid," RSC Advances, vol. 3, no. 2, pp. 513-519, 2013.

[19] Z. Abdullaeva, E. Omurzak, C. Iwamoto et al., "Onion-like carbon-encapsulated $\mathrm{Co}, \mathrm{Ni}$, and $\mathrm{Fe}$ magnetic nanoparticles with low cytotoxicity synthesized by a pulsed plasma in a liquid," Carbon, vol. 50, no. 5, pp. 1776-1785, 2012.

[20] E. Omurzak, T. Mashimo, S. Sulaimankulova et al., "Wurtzite-type ZnS nanoparticles by pulsed electric discharge," Nanotechnology, vol. 22, no. 36, article 365602, 2011.
[21] L. L. Chen, E. Omurzak, S. Takebe, C. Iwamoto, and T. Mashimo, "Graphitic carbon-coated $\mathrm{ZrC}$ - and Conanoparticles synthesized by pulsed plasma in liquid," Advanced Materials Research, vol. 236, pp. 1978-1982, 2011 a.

[22] L. Chen, C. Iwamoto, E. Omurzak et al., "Synthesis of zirconium carbide $(\mathrm{ZrC})$ nanoparticles covered with graphitic "windows" by pulsed plasma in liquid," RSC Advances, vol. 1, no. 6, pp. 1083-1088, 2011b.

[23] V. M. García-Orozco, C. Barrera-Díaz, G. Roa-Morales, and I. Linares-Hernández, "A comparative electrochemical-ozone treatment for the removal of phenolphthalein," Journal of Chemestry, vol. 2016, Article ID 8105128, p. 9, 2016. 

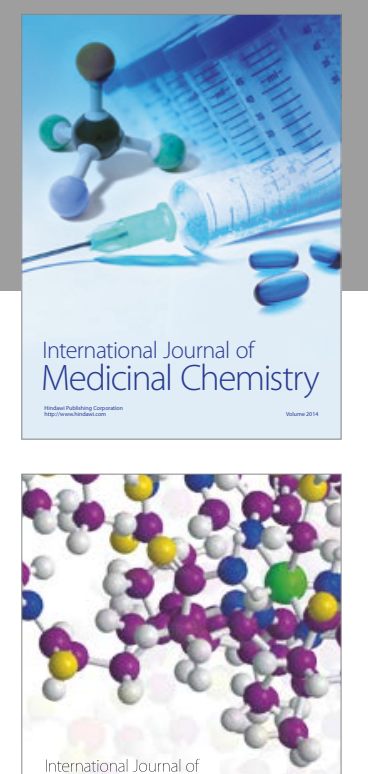

Carbohydrate Chemistry

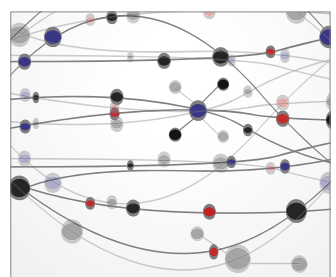

The Scientific World Journal
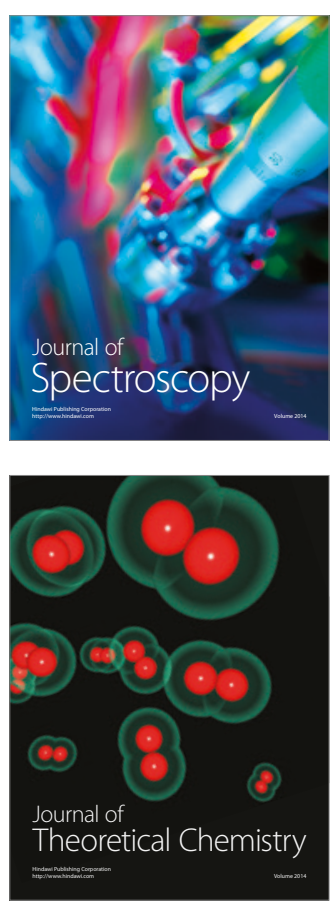
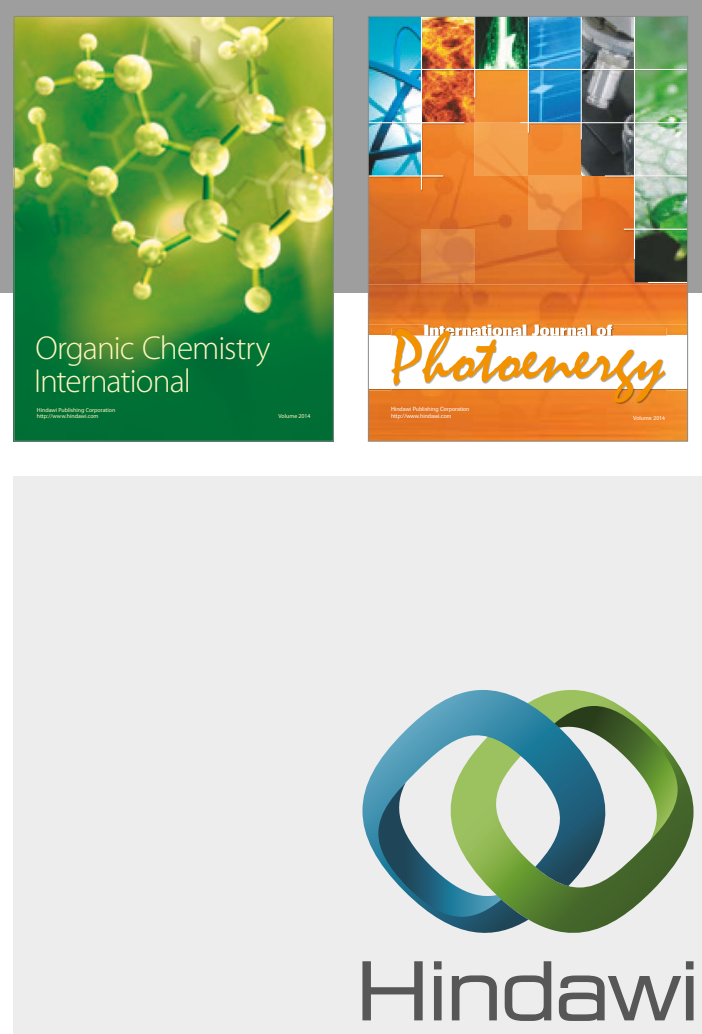

Submit your manuscripts at

https://www.hindawi.com

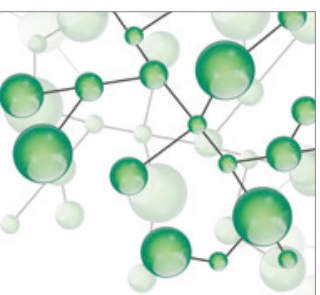

International Journal of

Inorganic Chemistry

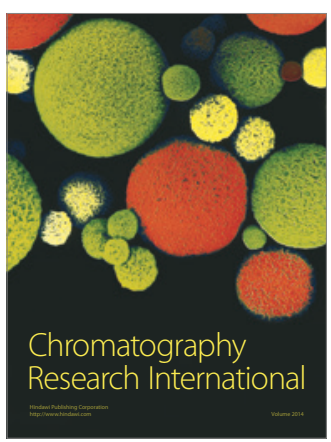

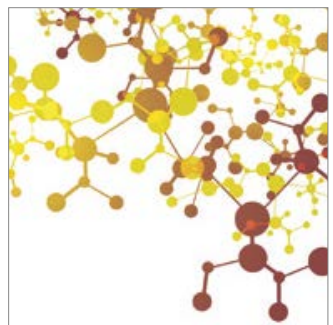

Applied Chemistry
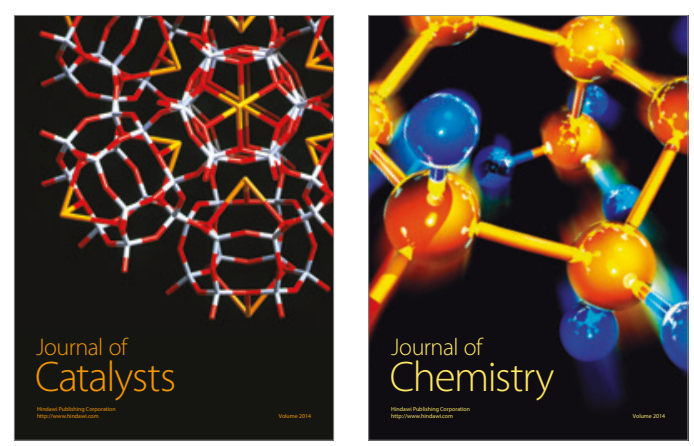
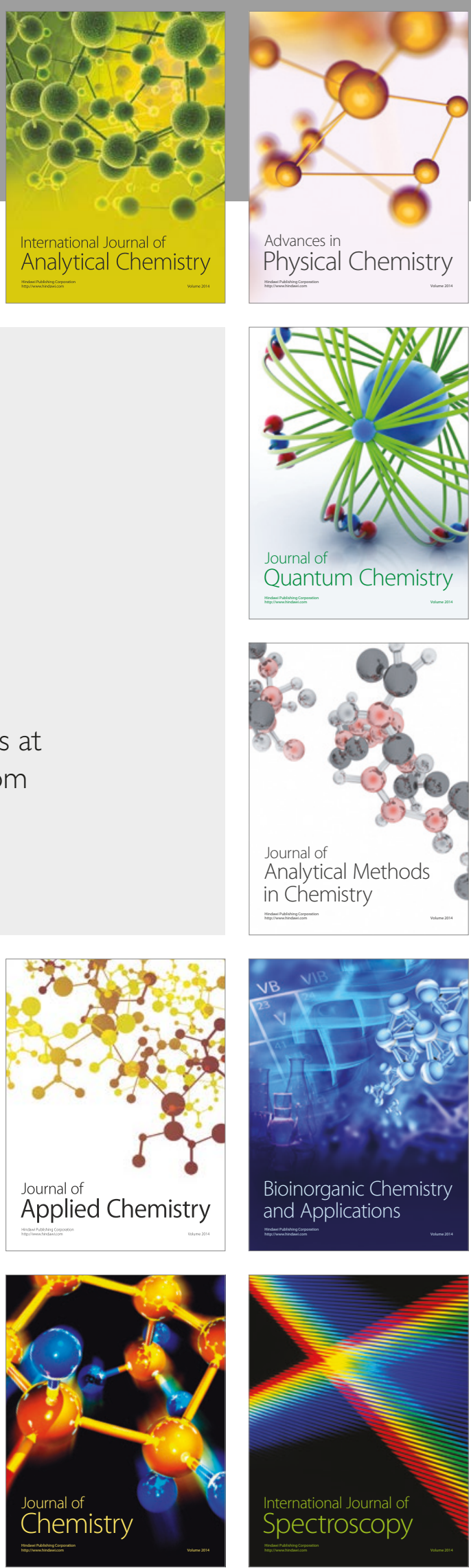